\title{
Apoyo social en la maternidad indeseada de estudiantes universitarias ecuatorianas: Análisis desde la perspectiva de género ${ }^{1}$ Social Support in unwanted maternity of ecuadorian undergraduate college students: Analysis from a gender perspective
}

\author{
Johanna Alexandra Reina-Barreto* \\ Cristina Alexandra Criollo-Espín** \\ Karina Fernández-D'Andrea**
}

\section{Resumen}

Existe una alta prevalencia de embarazos indeseados en jóvenes universitarias ecuatorianas. Esta investigación transversal de alcance correlacional se pregunta cómo ha sido posible conciliar ambos roles, de madre y universitaria, encontrando en el apoyo social percibido, la diferencia que permitió no abandonar sus estudios.

A partir de una muestra caracterizada socio-demográficamente, se analiza el apoyo social percibido por 50 madres universitarias de la sierra

\footnotetext{
${ }^{1}$ Este artículo de investigación se deriva de la estancia predoctoral de la primera autora, Financiado por la Beca Erasmus+ Internacional. Movilidad Doctoral 16-17 Universidad Complutense de Madrid - Universidad Técnica de Ambato. Algunos de sus resultados se presentaron en el III Congreso Internacional de Trabajo Social CIFETS 2018 Bilbao-España del 14 al 16 de noviembre de 2018.

* Trabajadora Social por la Universidad Colegio Mayor de Cundinamarca. Magíster en Trabajo Social Comunitario por la Universidad Complutense de Madrid. Actualmente doctoranda en Trabajo Social por la Universidad Complutense de Madrid. Madrid, España. Correo electrónico: joreina@ucm.es.

** Trabajadora Social por la Universidad Técnica de Ambato. Ambato, Ecuador. Correo electrónico: criscriollo93@gmail.com.

*** Trabajadora Social por la Universidad de Buenos Aires (UBA, Argentina). Magíster en Terapia Narrativa y Trabajo Comunitario por la Universidad de Extremadura (UEX) y en Intervención social en las sociedades del conocimiento por la Universidad Internacional de La Rioja (UNIR). Doctoranda en Trabajo Social en la Universidad Complutense de Madrid. Madrid, España. Correo electrónico: carinafe@ucm.es.

Universidad del Valle. Cali, Colombia. Recibido: 30/07/2018 Aprobado: 16/10/2018 ISSN: 0122-1213 ISSN-e: 2389-993X Doi: 10.25100/prts.v0i27.6745
} 
ecuatoriana. Se encontró que el $80 \%$ del total de madres, inició la maternidad cursando estudios como consecuencia de embarazos no deseados. El 44,9\% interrumpió los estudios por esta causa y el apoyo social fue el facilitador principal para continuar el doble rol, de madre y estudiante. Asimismo emergen diferencias significativas entre las solteras y las casadas/unidas respecto a todas las variables de estudio. Se encontraron asociaciones estadísticamente significativas entre haber contraído matrimonio a causa del embarazo y continuar casada así como la percepción de apoyo por la pareja y tiempo de acompañamiento en la maternidad. Los resultados evidencian la importancia de diseñar programas, con perspectiva de género, que faciliten la conciliación y minimicen el riesgo de deserción universitaria.

Palabras clave: Maternidad; Apoyo Social; Deserción Escolar; Perspectiva de género; Trabajo Social en Educación Superior; Maternidad indeseada.

\section{Abstract}

There is a high prevalence of undesired pregnancies among young Ecuadorian university students. This correlational cross-sectional research inquires how it has been possible to reconcile both roles, that of a mother and of a student. The findings show that the perceived social support marks the difference that allowed women not to abandon their studies.

Based on a sample, characterized socio-demographically, of 50 women who are both mothers and undergraduate college students in the central zone of Ecuador, the study analyzes the structural social support and the functional social support.

It was found that $80 \%$ of mothers started motherhood during the course of their studies and as a product of undesired pregnancies. $44.9 \%$ interrupted their studies due to undesired maternity and social support was the main factor which impulse them to continue the double role of mother and student. Significant differences also emerged between single and married/united women with respect to all the variables in the study. The chi-square reveals a statistically significant association between getting married as a result of pregnancy and staying married, and also between 
the perception of support of the partner and the time of accompaniment in maternity.

The results showed the importance of designing specific programs with a gender perspective that facilitate the conciliation of both roles and minimize the risk of dropping out of university.

Keywords: Motherhood; Social support; School Dropout; Gender perspective; Social Work in Higher Education; Unwanted motherhood.

Sumario: 1. Introducción; 2. Metodología; 3. Muestra; 3.1. Características sociodemográficas de la muestra 4. Resultados; 5. Discusión; 6. Conclusiones; 7. Referencias bibliográficas.

\section{Introducción}

La tasa de maternidad en jóvenes ecuatorianas lejos de disminuir va en aumento en comparación con otros países sudamericanos. Para comprobarlo se ha comparado el porcentaje de madres entre 18 y 19 años en Ecuador, Colombia, Venezuela, Bolivia, Perú, Chile, Brasil, Paraguay, Uruguay y Argentina; a partir de los reportes y estadísticas de género de la CEPAL (sf).

El análisis demuestra que Ecuador registra la tasa de maternidad más alta del cono sur y es el único con una tendencia sostenida de incremento de la misma. Sí en el año 1990 el 23,7\% de mujeres entre 18 y 19 años eran madres, en 2010 lo era un 28,6\% CEPAL (sf).

A partir del cruce de datos del censo de la población ecuatoriana en 2017 y el registro de nacidos vivos del mismo año, se encuentra que 31.274 mujeres de ese grupo etario ya son madres, es decir más de una de cada tres ecuatorianas entre 18 y 19 años (33,9\%) (INEC, sf; 2017). Si se suman los 157 casos de defunciones fetales, la prevalencia llega al 34\% (INEC, 2017). Se desconoce el número de abortos clandestinos practicados, lo que permite sospechar que el porcentaje de embarazos en jóvenes es todavía mayor.

El mismo análisis en los países latinoamericanos mencionados, señala que el porcentaje de maternidad varía en más de 11 puntos y va desde 
el $17,3 \%$ en Uruguay hasta el $28,6 \%$ en Ecuador. La media de la región indica que una de cada cinco mujeres entre 18 a 19 años es madre $(22,1 \%$ SD 3,1). Estas cifras contrastan abruptamente con el 3,4\% registrado en el mismo grupo poblacional de mujeres en España (Observatorio de Igualdad de Género de América Latina y el Caribe, sf), donde la media de edad para inicio de la maternidad se sitúa en los 32 años (INE, 2018).

Desde la perspectiva de Género se aportan elementos que permiten analizar las formas diferenciadas de socialización entre hombres y mujeres. En ellas se han exaltado los papeles reproductivos de madre y esposa como los principales roles femeninos. A través de éstos se medirá su éxito, realización y adultez (Puyana-Villamizar y Mosquera-Rosero, 2005).

Ambos roles se sustentan en estereotipos transformados en axiomas que exaltan las prácticas tradicionales y definen el modelo de "buena madre" y que por otra parte son el sustrato de las actuales, pero invisibilizadas, presiones sociales ejercidas hacia la mujer (Amantze, 2015; Arvelo, 2004; Pacheco-Carpio, Cabrera-Albert, Mazón-Hernández, González-López y Bosque-Cruz, 2014; Palomar-Verea, 2004; Rodríguez-Otero y MancinasEspinoza, 2016).

Este modelo hegemónico, identifica mujer con madre e implica una serie de características atribuidas a las mismas: ser sumisa, inferior, débil, asexual, sacrificada, abnegada, dedicada a la casa y a la crianza de los hijos, ser cuidadora y ser para otros (Cristiani et al., 2015; Facio, 2002; Lagarde 1990; Sánchez-Benítez, 2016). Imagen que es reforzada socialmente por instituciones como la familia, la escuela, el grupo de amigos, la comunidad o la religión, entre otros (Ferrer-Pérez y Bosch-Fiol, 2013; García-Selgas y Casado-Aparicio, 2010; Maeso-Fernández et al., 2015; Rodríguez-Otero y Mancinas-Espinoza, 2016).

En Ecuador, comienza a darse la redefinición de los roles femeninos a partir de la segunda mitad del siglo XX, con la conquista del espacio público por parte de las mujeres (Mora-Bleda, 2013). Primero con el reconocimiento de sus Derechos Sociales durante la Revolución Juliana en 1925 (Paz y Cepeda, 2002) o de sus Derechos Civiles en 1948 (Pizani, 2009) y luego con su incorporación masiva a la educación y al trabajo. 
Pero, lo cierto es que la maternidad continúa incorporada con mucha fuerza a la identidad femenina en la actual sociedad ecuatoriana del siglo XXI.

Las jóvenes ecuatorianas se ven impelidas a responder a ambos roles, el emancipador y el ejercicio tradicional de la maternidad consecuencia en muchos casos de embarazos no planificados ni deseados, pero llevados a término. En el contexto universitario esta situación impacta profundamente en la universitaria, su familia, la pareja y sus amigos; e influye por tanto, en las esferas académica, emocional y social (Domínguez-Aguirre, MartínezPeralta, Herrera-Barragán y Rincón-Aguirre, 2015; Guzmán-Gómez, 2017; Texis y Jiménez, 2016). Además, en algunos estudios se ha documentado el abandono de las parejas, viéndose obligadas a llevar en solitario la crianza (madresolterismo) (Estupiñán-Aponte y Rodríguez-Barreto, 2009; ZapataPosada, Castro-Rodelo y Agudelo-Bedoya, 2016).

No obstante, estas consecuencias tienden a variar según el apoyo social, en adelante AS, que las estudiantes perciben de sus fuentes o redes de apoyo informal (familia, amigos, compañeros y vecinos) y formal.

El apoyo social (AS) es un constructo multidimensional que puede entenderse, entre otras, desde dos perspectivas principales: la estructural y la funcional. La primera, quién provee el apoyo, analiza la existencia, estructura y densidad de las potenciales redes de apoyo, siendo éstas informales o formales. Las informales hacen referencia a las redes primarias como son la familia, los/as amigos/as, compañeros/as de estudio o de trabajo; las formales abarcan las instituciones o entidades conformadas por la sociedad y/o el estado que tienen por finalidad ofrecer algún servicio o atender una demanda o necesidad social (Barrón, 1996; Díaz, 1987; Feldman et al., 2008; Hombrados-Mendieta, 2013; Montes de Oca, 2000). La perspectiva funcional estudia a su vez tanto la índole del apoyo (percibido y/o recibido) como los diferentes tipos específicos de AS, que pueden ser de tipo material o instrumental, informacional, emocional o económico (Barrera, 1986; Barrón, 1996; Díaz, 1987; Montes de Oca, 2000).

Pero las mujeres pueden recibir apoyo social y no percibirlo. Por ello en esta ocasión se aborda únicamente el "apoyo social percibido". 
Algunas investigaciones han hecho ya referencia directa e indirecta a la necesidad de soporte que las universitarias requieren de sus redes sociales para la conciliación del doble rol. Otros estudios confirman que la posibilidad de encontrar apoyo social es el factor desencadenante que facilita que la nueva condición de maternidad no se convierta en un obstáculo mayor en su preparación académica (Castillo-Sánchez, 2015; Domínguez-Aguirre et al., 2015; Lozano-Cabezas, Iglesias-Martínez y Alonso-Sanz, 2016) y que el papel de la abuela es clave en esa conciliación (Zapata-Posada et al., 2016).

La Red Social proporciona el marco estructural que hace posible a la persona acceder al apoyo aunque, como argumenta Díaz (1987), recibir o no ayuda también depende de que la red valore que la persona merece recibir el apoyo que demanda (Díaz, 1987, p. 137).

Varios/as autores/as describieron que la primera red de apoyo informal para estas mujeres era su familia de origen, especialmente la madre quien les proveía diferentes tipos de AS: material, económico, y emocional (Cimino-Sandoval, Durán-Sepúlveda, Herbage-Amaro, Palma-Jara, y Roa-Vergara 2014; Domínguez-Aguirre et al., 2015; Estupiñán-Aponte y Rodríguez-Barreto, 2009; Preciado-Cortés, Acuña-Cepeda y GarcíaRivera, 2005; Zapata-Posada et al., 2016).

Pareciera entonces que el AS es una variable que contribuye en la conciliación de ambos roles y que la familia juega un papel decisivo en este tema.

Respecto al apoyo formal, en el ámbito universitario, la literatura informa sobre la necesidad de facilitar la conciliación de ambos roles (universitaria y madre) por medio de programas o acciones institucionales (Estupiñán-Aponte y Rodríguez-Barreto, 2009; Estupiñan-Aponte y Vela, 2012).

Otros estudios indican que el AS que brinda la red de apoyo formaluniversidad es percibido como algo negativo por las madres universitarias debido al rechazo hacia ellas, por parte de docentes y compañeros de clase, relacionado con la condición de maternidad de la estudiante y su rendimiento académico (Domínguez-Aguirre et al., 2015; EstupiñánAponte y Rodríguez-Barreto, 2009; Estupiñán-Aponte y Vela, 2012). 
Siendo una investigación desde Trabajo Social, interesa conocer si la existencia de Trabajadoras/es Sociales en los departamentos de bienestar universitario, pudieran aportar a dicha conciliación desde su práctica profesional. Dadas las importantes implicaciones de esta doble condición y el riesgo de abandono de los estudios, el tema recobra especial importancia para el Trabajo Social en el ámbito de la educación superior en Ecuador.

En resumen, esta investigación transversal de alcance correlacional, pretende dar cuenta del apoyo social desde las perspectivas estructural y funcional (solo en su dimensión apoyo percibido) en relación al ejercicio de la maternidad en estudiantes universitarias ecuatorianas, incorporando al análisis las variables sociodemográficas de la mujer, de su pareja y su condición socioeconómica.

La perspectiva de género, retoma los elementos teóricos antes mencionados para identificar qué lógica subyace y cómo intersectan dos identidades, el ser madre y el ser estudiante en una población con la mayor prevalencia de embarazos indeseados en América Latina, puesto que pareciera a priori que son proyectos vitales contradictorios.

\section{Metodología}

La investigación fue llevada a cabo en dos etapas. En la primera se realizó una exhaustiva revisión bibliográfica con los descriptores de búsqueda: Apoyo Social, Estudiante, Madre y Ecuador, en las bases de bases de datos de Dialnet, Latindex y Google Académico. Como resultado preliminar, se diseña el cuestionario ad hoc de 49 preguntas cerradas y una pregunta abierta que abarcaban las condiciones sociodemográficas tanto de la mujer como de su pareja y el AS estructural y funcional relacionado con el doble rol que desempeñan: madre y estudiante.

En una primera fase se aplicó una prueba piloto con 11 madres universitarias y se verificó la pertinencia y exhaustividad del instrumento para los fines propuestos.

En la segunda etapa se obtuvo autorización del decanato para contactar con las estudiantes madres que conforman la unidad de análisis. En total 80 mujeres que estudian en facultades de Trabajo Social, Comunicación 
Social y Derecho, en una universidad de la sierra ecuatoriana, pública y gratuita.

En cada curso se informó sobre el objetivo de la investigación y se solicitó la participación voluntaria y sin retribución económica de las estudiantes madres.

El trabajo de campo se realizó durante los meses de abril y mayo de 2018 en horario diurno. El cuestionario ha sido auto-completado por las estudiantes y son ellas quienes informan sobre sus parejas. El análisis de datos se realizó con el programa SPSS V.22 y Microsoft Excel. Todos los contrastes estadísticos se realizan mediante el contraste de chi cuadrado.

En la depuración de la base de datos, se ha identificado un cuestionario que no ha sido completado en su totalidad, sin embargo, para conservar la mayor cantidad de información obtenida, se ha decidido tener en cuenta las respuestas que han sido suministradas. Por tal razón, en algunos análisis de contraste estadístico, la muestra se reduce a 49 informantes mientras que otros contrastes informan de la totalidad de la muestra estudiada.

\section{Muestra}

La unidad de observación corresponde a una muestra no probabilística de 50 mujeres que responden a la doble condición de ser madres y estudiantes universitarias y que cursan actualmente estudios. La prevalencia global de maternidad (número de madres estudiantes respecto del total de mujeres estudiantes) para el conjunto de mujeres estudiantes de esa facultad fue del 13,9\%. La prevalencia de maternidad por cada carrera, se ha calculado con base en la información actualizada a Mayo de 2018 ${ }^{2}$. En el caso de Trabajo Social la prevalencia de maternidad es del 21\%; en la carrera de Comunicación Social es del 9,5\% y en Derecho del 7,2\%. Se ha calculado la tasa de respuesta de la muestra a partir de los datos de madres universitarias de toda la facultad: $62,5 \%$. Respecto a la representatividad por carrera en la muestra, un $84 \%$ de mujeres estaban cursando estudios en Trabajo Social, un $12 \%$ estudios en Comunicación Social y un $4 \%$ estudiando Derecho. ${ }^{3}$

\footnotetext{
${ }^{2}$ Datos facilitados por la secretaría académica

${ }^{3}$ La baja participación de las madres que cursan estudios en Derecho en la muestra final, se entiende en tanto la jornada de estudio de esta carrera es predominantemente nocturna Prospectiv a. Revista de Trabajo Social e inter vención social No. 27, enero-junio 2019: pp. 107-137 (c) (1) () @ 


\subsection{Características sociodemográficas de la muestra}

La edad de las madres de la muestra, está comprendida entre los 19 y 38 años, siendo la media 23,9 años (SD 3,8 años). El 27,1\% tiene 23 años, seguido del $16,7 \%$ con 25 años. Nueve de cada diez mujeres se autoidentifica como mestiza $(91,8 \%)$ y el $8,2 \%$ como mujer indígena. Una de cada tres proviene de entornos rurales $(32,7 \%)$ y el $14 \%$ ha cambiado su residencia por los estudios.

Dado que la maternidad es sobrevenida durante la universidad, es lógico que la gran mayoría tenga sólo un hijo/a $(85,7 \%)$ con pocos años de vida (46,9\% tiene menos de dos años y otro $32,7 \%$ tiene entre 3 y 5 años). Se registra también un 6,1\% de segundos embarazos, de los cuales el 33\% vuelve a ser un embarazo no planificado.

Sus familias se conforman por un promedio de 4,37 personas/hogar (SD $1,5)$ y tienen 2,3 hermanos/as (SD 1,6). El 55,1\% vive con su familia de origen $(32,7 \%$ estructura nuclear, $18,4 \%$ monomarentales - uniparentales y $4,1 \%$ extensa) y el otro $44,9 \%$ vive con su familia propia, es decir la que ellas han conformado con sus parejas o solas $(30,6 \%$ estructura nuclear, $10,2 \%$ extensa y $4,1 \%$ monomarental).

La cabeza de hogar es masculina en el $55,1 \%$ de todas las familias de la muestra y femenina en el $46 \%$. Cuando en la familia de origen de la estudiante vive un sólo progenitor, éste es mujer, por ello, la madre es la cabeza de hogar en el $88,9 \%$ de esos hogares.

En relación a su estado civil, el 42,9\% son solteras y el 57,1\% conviven con su pareja (bajo esta categoría se agrupará al 38,8\% casadas y el 18,3\% de mujeres que conviven en uniones libres). Entre las solteras, el 57,1\% informa no mantener relación sentimental actual; de las solteras que sí la mantienen, el 66,7\% lo hace con una persona diferente al padre biológico de su hijo/a.

Por otra parte, y en relación al nivel educativo de la pareja, uno de cada cuatro tiene estudios universitarios sin terminar (24\%) y uno de cada tres cursa estudios actualmente (32\%); el 18\% finalizó la secundaria, el 14\%

y los conversatorios se realizaron en horario diurno conforme a la autorización conseguida del decanato. 
estudios superiores completos, el $8 \%$ estudios técnicos y el $4 \%$ posgrado. Es decir, igual o menor nivel de estudios.

Analizando las variables socioeconómicas, el 71,4\% \% se auto-clasifica dentro del estrato socioeconómico medio y el $28,6 \%$ en el estrato mediobajo. Una de cada dos informa de vivienda propia $(53,1 \%)$; una de cada cinco vive de alquiler $(22,4 \%)$ y una de cada cuatro en viviendas familiares o prestadas $(24,5 \%)$. La mayoría habita casas $(73,5 \%)$, conforme a la estructura urbanística predominante de la zona. El 6,1\% informaron habitar viviendas precarias (cuartos de inquilinatos o casas media agua ${ }^{4}$ según clasificación del INEC, 2017).

Respecto a la situación laboral de las mujeres, sin discriminar solteras o que conviven en pareja, la gran mayoría de ellas todavía no tienen experiencia laboral $(85,7 \%)$. Una de cada cuatro está buscando empleo $(24,5 \%)$, el 8,2\% tienen trabajos ocasionales y sólo el 6,1\% tiene trabajo formal y de ellas el 66,7\% tiene una experiencia de 7-8 años (son las mujeres con mayor edad).

En cuanto a la información laboral de sus parejas y/o padres de sus hijas/ os, las mujeres de la muestra informaron una tendencia inversa. Siete de cada diez hombres trabaja (69,4\%). El 12,2\% no trabaja y de éstos el 6,7\% busca empleo actualmente. En el 18,4\% de los casos la mujer desconoce la condición laboral del padre de su hija/o aun estando en pareja.

Entre las parejas que trabajan, uno de cada dos tiene empleo formal asegurado al $\operatorname{IESS}^{5}$ o al $\operatorname{ISSPOL}^{6}(57,6 \%)$, uno de cada tres trabaja ocasionalmente $(30,3 \%)$ y el $9,1 \%$ tiene un empleo precario. Siete de cada diez trabaja 40 h o más/semana $(69,7 \%)$, otro $18,2 \%$ menos de $20 \mathrm{~h} /$ semana y un $12,1 \%$ entre $21-30 \mathrm{~h} /$ semana.

En conclusión, el perfil de la muestra se caracteriza por tener una edad media de 23-24 años. Se autodenomina como mestiza (9 de cada 10), con mayoría de un hijo/a $(85,7 \%)$, con más de la mitad conviviendo en pareja

\footnotetext{
${ }^{4}$ Vivienda de un único nivel que construye el techo con una caída de aproximadamente $45 \%$ de inclinación, con paredes de adobe, bloque, ladrillo o madera y techos de paja, zinc, asbesto, etc.

${ }^{5}$ IESS: Instituto Ecuatoriano de la Seguridad Social.

${ }^{6}$ ISSPOL: Instituto de Seguridad Social de la Policía Nacional de Ecuador.
} 
$(57,1 \%)$ y la otra mitad solteras $(42,9 \%)$. Más de la mitad $(57,1 \%)$ no tiene relación sentimental con el padre de su hija/o y de ellas, sólo el 28,7\% tiene relación con un hombre (diferente al padre biológico). La mayoría se posiciona en el estrato socioeconómico medio (71\%). Una de cada cinco vive en alquiler $(22,4 \%)$. A nivel educativo, las parejas tienen menor formación que ellas (uno de cada cuatro tiene estudios universitarios sin terminar), pero cuando se describe la situación laboral, la tendencia es inversa, ya que mientras la mayoría de parejas trabajan $(69,4 \%)$, de las mujeres universitarias el $85,7 \%$ no tiene experiencia laboral.

\section{Resultados}

Respecto a la alta natalidad de jóvenes universitarias de la sierra ecuatoriana, se encuentra que la gran mayoría han sido embarazos no deseados para cuatro de cada cinco universitarias, idéntica al porcentaje de embarazos no planificados (79,6\%). Además tres de cada cinco mujeres tuvo miedo de comunicar su embarazo (62\%) y una de cada cuatro informó pensamientos de aborto (26\%). De aquí se concluye que se trate de maternidades no deseadas.

La condición de convivencia o no en pareja fue determinante en la formación de dos grupos con diferencias sociodemográficas, económicas y de AS. El 46,4\% del total de la muestra se casó a causa del embarazo, al cruzar este dato con quienes informan convivencia con pareja el porcentaje llega al 92,9\% como se muestra en la tabla 1. La relación matrimonio por embarazo y convivencia actual es estadísticamente significativa mediante contraste chi cuadrado $\mathrm{X}^{2}=(1 \mathrm{~N}=49)=9,692, \mathrm{P}<0,05$. 
Tabla 1. Convivencia actual *Se casó o unió por motivo de su embarazo tabulación cruzada

\begin{tabular}{|c|c|c|c|c|}
\hline \multicolumn{5}{|c|}{ \% dentro de Se casó o unió por motivo de su embarazo } \\
\hline & & \multicolumn{2}{|c|}{$\begin{array}{c}\text { Se casó o unió } \\
\text { por motivo de su } \\
\text { embarazo }\end{array}$} & \multirow[b]{2}{*}{ Total } \\
\hline & & No & SI & \\
\hline \multirow[t]{2}{*}{$\begin{array}{c}\text { Convivencia } \\
\text { actual }\end{array}$} & $\begin{array}{c}\text { Sin convivencia actual } \\
\text { (soltera) }\end{array}$ & $55,9 \%$ & $7,1 \%$ & $41,7 \%$ \\
\hline & $\begin{array}{c}\text { Convivencia actual } \\
\text { (casada o unión libre) }\end{array}$ & $44,1 \%$ & $92,9 \%$ & $58,3 \%$ \\
\hline \multicolumn{2}{|c|}{ Total $(\mathrm{N}=50)$} & $100,0 \%$ & $100,0 \%$ & $100,0 \%$ \\
\hline
\end{tabular}

Fuente: elaboración propia.

Por otra parte, cuando se analiza el estado civil de la mujer y quién es la cabeza de hogar, la relación de ambas variables es estadísticamente significativa mediante contraste chi cuadrado $\mathrm{X}^{2}=(1 \mathrm{~N}=50)=7,039, \mathrm{P}<0,05$. Lo que significa que estar soltera o convivir en pareja se relaciona con el hecho de que el hogar sea dirigido por una mujer o un hombre. En este sentido se encontró que cuando la cabeza de hogar es femenina, la hija universitaria es soltera en mayor proporción, vive con su familia de origen $y$ tiende a ser un hogar monomarental.

En cuanto a las características socioeconómicas de los hogares, como se observa en la tabla 2 , el cruce de estrato socioeconómico y tipología de vivienda revela que sólo habitan viviendas precarias quienes se autoclasificaron en el estrato más bajo, el 21,4\% de universitarias. Esta relación es estadísticamente significativa mediante contraste chi cuadrado $\mathrm{X}^{2}=(2 \mathrm{~N}=49)=8,085, \mathrm{P}<0,05$. y sugiere que la autopercepción de mayor precariedad económica se evidencia en las condiciones de la vivienda y ésta, por tanto, constituye un indicador de este nivel socioeconómico para valorar la potencial provisión de apoyos económico. 
Tabla 2. Auto identificación en estrato socioeconómico *Tipo de vivienda según INEC - Ecuador tabulación cruzada

\begin{tabular}{|c|c|c|c|c|c|}
\hline \multicolumn{6}{|c|}{$\%$ dentro de auto-clasificación en estrato socioeconómico } \\
\hline & & \multicolumn{3}{|c|}{ Tipo de vivienda según INEC } & \multirow[b]{2}{*}{ Total } \\
\hline & & Casa & Departamento & $\begin{array}{l}\text { vivienda } \\
\text { precaria }\end{array}$ & \\
\hline \multirow{2}{*}{$\begin{array}{c}\text { Estrato } \\
\text { socioeconómico }\end{array}$} & media & $77,1 \%$ & $22,9 \%$ & & $100,0 \%$ \\
\hline & media-baja & $64,3 \%$ & $14,3 \%$ & $21,4 \%$ & $100,0 \%$ \\
\hline \multicolumn{2}{|c|}{ Total $(\mathrm{N}=50)$} & $73,5 \%$ & $20,4 \%$ & $6,1 \%$ & $100,0 \%$ \\
\hline
\end{tabular}

Fuente: elaboración propia

Como ya se mencionó, del cruce de las variables sociodemográficas y un análisis mediante el contraste de chi cuadrado, emergen dos grupos con características sociodemográficas diferenciadas. Con este criterio se presenta la siguiente caracterización:

\subsection{Madres universitarias solteras: Ningún embarazo fue planificado,} existió miedo de comunicarlo y pensamientos de aborto. Provienen de más familias unimarentales con peores condiciones socioeconómicas. Se ven abocadas a asumir el embarazo y la crianza en solitario, sólo una de cada tres mujeres es acompañada por su pareja en la crianza hasta la actualidad

Prácticamente todas las universitarias solteras iniciaron la maternidad durante el transcurso de sus estudios $(95,2 \%)$ y tienen un hijo/a únicamente. Ninguna había planificado su embarazo y cuatro de cada cinco, tenía miedo de comunicarlo (81\%). El 28,6\% tuvo pensamientos de aborto, lo cual evidencia, como se ha descrito, que la maternidad es una condición no deseada por estas mujeres.

Se ubican a sí mismas en mayor proporción dentro del estrato medio ( $85,7 \%$ vs. $60,7 \%$ unidas), a pesar de ser menos propietarias de vivienda y habitar más viviendas precarias $(4,8 \%)$. Dos de cada cinco de ellas proviene de una familia con un único progenitor $(42,9 \%)$ donde la cabeza de hogar es femenina en tres de cada cinco hogares $(66,7 \%)$, y son familias algo más numerosas respecto a las mujeres que conviven en pareja $(4,6$ personas/flia). 
En relación a la situación laboral, casi tres de cada cinco universitarias no tiene experiencia laboral $(57,1 \%)$, y un $28 \%$ está buscando empleo. El $14,3 \%$ ha tenido trabajos ocasionales.

El 38\% de universitarias no sabe o no informa acerca de sus exparejas y la ruptura total del vínculo del padre con sus hijo/a se verifica con el análisis de otras variables. Esta condición impacta en las potenciales redes de apoyo con las que pueden contar las madres universitarias solteras, pues podría significar una menor red a la que poder demandar diferentes tipos de apoyo.

El 23,8\% de las exparejas tiene estudios universitarios incompletos, otro $14,3 \%$ culminó la universidad y un $4,8 \%$ educación de ciclo medio, el $33,1 \%$ continúa estudios.

En relación a la situación de empleo de los padres de sus hijas/os, dos de cada cinco trabaja $(42,9 \%)$ y de ellos, uno de cada cinco tiene un empleo formal (23,8\% vs. 14,3\% trabajo ocasional). El 47\% de mujeres desconoce este dato. El 100\% de mujeres solteras no recibe apoyo económico del padre de su hijo/a, lo cual revela que la carga económica para la crianza y manutención es asumida en un porcentaje muy importante por otras personas diferentes al padre.

Sólo una de cada tres universitarias de este grupo es acompañada en el proceso de crianza por el padre de su hija/o; por tanto la mayoría asumen un modelo de maternidad diferente al modelo tradicional madre-esposa, denominado por algunas/os autores/as madresolterismo.

\subsection{Madres universitarias en convivencia (en pareja): La mayoría también fueron embarazos no planificados, provienen más de familias nucleares y se casan porque se embarazan, aunque una de cada tres continúa viviendo con su familia de origen. Sus parejas tienen mayores niveles formativos y la mayoría de ellos trabaja}

Para el caso de universitarias que informaron convivencia en pareja, se encontró que casi siete de cada diez iniciaron su maternidad durante el transcurso de estudios $(67,9 \%)$, producto de embarazos no planificados $(64,3 \%)$. Además dos de cada cinco mujeres tuvo miedo de contarlo $(46,4 \%)$ y una de cada cinco informó pensamientos de aborto $(21,4 \%)$. Estos datos

Prospectiv a. Revista de Trabajo Social e inter vención social No. 27, enero-junio 2019: pp. 107-137 (c) (i) () () 
sugieren que en este grupo, la maternidad tampoco fue una condición deseada o planificada para la mayoría de universitarias. La convivencia en pareja inicia después de conocerse que la mujer se encuentra en estado de embarazo. El emparejamiento con el padre de su futura/o bebé se da sobre todo mediante matrimonio católico $(67,9 \%)$ y en mucha menor proporción mediante convivencia sin reconocimiento legal, es decir la unión de hecho $(32,1 \%)$. El alto porcentaje de matrimonios católicos se entiende por el fuerte arraigo cultural hacia este tipo de religión y por la idea de que los/ as hijos/as se deben concebir dentro del mismo; supeditando cualquier otro proyecto vital femenino a su nueva condición de madre-esposa.

La relación entre matrimonio por embarazo y convivencia actual es estadísticamente significativa mediante contraste chi cuadrado $\mathrm{X}^{2}=(1$ $\mathrm{N}=49$ ) $=9,692, \mathrm{P}<0,05$. Es decir, en este grupo, las mujeres más jóvenes se casan porque se embarazan, conforme al estereotipo ideal de familia patriarcal.

Ellas se auto-clasifican más en el estrato medio-bajo respecto a las solteras $(39,3 \%$ vs. $14,3 \%)$ y aunque cabría esperar que todas convivan con sus parejas, resulta que tres de cada diez vive con su familia de origen, $(32,1 \%)$ donde el cabeza de hogar es mayoritariamente masculino $(71,4 \%)$.

Sus hogares son menos numerosos respecto a los hogares de las madres universitarias solteras (4,18 personas/flia), lo cual es lógico teniendo en cuenta que ellas son en mayor proporción hijas únicas $(10,7 \%$ vs 5\% solteras).

De acuerdo a la condición laboral, las madres universitarias de este grupo tienen menor experiencia que las solteras (64,3\% vs. 57,1\% respectivamente); pero también aquí se ubican todas aquellas mujeres que tienen un trabajo formal $(10,7 \%)$. Esta situación se entiende porque se trata de las mujeres con mayor edad en la muestra (36 a 38 años). Además, el reporte de trabajo ocasional $(3,6 \%)$ y búsqueda de empleo $(21,4 \%)$ también es inferior respecto las solteras.

En el caso de sus parejas, nueve de cada diez trabaja $(89,3 \%)$, uno de cada dos tiene empleo formal (50\%) y otro 35,7\% trabajo precario (ocasional o sin cotizar a la seguridad social). Dos de cada cinco trabaja jornada legal completa (42,9\%) y otro 17,9\% lo hace más de 40h/semana. El 
cruce de variables demuestra que cuando ellos trabajan, ellas tienden más a abandonar sus estudios. Sus parejas además tienen niveles formativos más altos (desde técnico hasta posgrado $57,1 \%$ ) y uno de cada tres continúa estudios $(32,1 \%)$.

\subsection{Apoyo Social Percibido: Perspectiva funcional y perspectiva estructural}

Respecto a la perspectiva funcional del AS, se indaga acerca de la provisión de apoyo de tipo económico (para sufragar gastos de estudios y de crianza), apoyo material (cuidado de hijos/as) y apoyo emocional (acompañamiento en gestación, puerperio e inicio de maternidad y comprensión por la situación de maternidad).

Se preguntó de qué red obtenía los tipos de apoyo mencionados, pudiendo identificar a la red de estructura informal (pareja, familia de origen, familia propia ${ }^{7}$, amistades) $y / o$ a la red de estructura formal (guarderías, universidad).

Durante el embarazo, cuatro de cada cinco universitarias percibió apoyo emocional de su pareja $(83,7 \%)$ y el $16,3 \%$ informó abandono del padre del bebé desde el inicio de la gestación, por lo que no recibió apoyo emocional.

Una de cada cinco mujeres $(20,4 \%)$ no informa apoyo emocional de su pareja durante el periodo de puerperio e inicio de la maternidad. Otro $16,7 \%$ reportó que el acompañamiento duró máximo, el primer año de vida del hijo/a. Esto significa que llegado el momento de ejercer la paternidad, más hombres abandonaron a sus hijos/as, abocando a la mujer a asumir en solitario toda la carga de la crianza en condiciones de dependencia económica, como indican los datos relativos a su situación laboral.

Sólo tres de cada cinco universitarias, informaron sentirse acompañadas por el padre de su hijo/a hasta la actualidad (63,3\%). Al cruzar ambas variables se encuentra que el $70 \%$ de quienes no percibieron apoyo por su pareja durante la gestación tampoco fueron acompañadas en el periodo de

${ }^{7}$ Se entenderá como familia propia la sumatoria de todos casos en los que ella vive con su pareja, bien sea con una estructura de familia nuclear (pareja e hijos/as), o extensa (pareja, hijas/os y otros familiares de su pareja).

Prospectiv a. Revist a de Trabajo Social e inter vención social No. 27, enero-junio 2019: pp. 107-137 (c) (1) () () 
puerperio y maternidad, como se ve en la tabla 3 . El contraste chi cuadrado revela que la relación entre percepción de apoyo durante el embarazo y el tiempo de acompañamiento de la pareja es estadísticamente significativa $\mathrm{X}^{2}=(2 \mathrm{~N}=49)=26,580, \mathrm{P}<0,05$. Otras fuentes de AS emocional citadas fueron provistas por la familia de origen $(36,7 \%)$, la dupla familia origen y propia $(32,7 \%)$, la familia propia $(16,3 \%)$ y las amistades $(14,3 \%)$.

Tabla 3. Apoyo emocional pareja durante periodo de gestación*Tiempo acompañamiento pareja en periodo de puerperio y maternidad tabulación cruzada

\begin{tabular}{cccccc}
\hline \% dentro de Tiempo acompañamiento pareja en periodo de puerperio y maternidad \\
\hline & \multicolumn{4}{c}{$\begin{array}{c}\text { Tiempo acompañamiento pareja en } \\
\text { periodo de puerperio y maternidad }\end{array}$} & \\
\cline { 2 - 5 } & & $\begin{array}{c}\text { No la } \\
\text { acompañó }\end{array}$ & $\begin{array}{c}\text { Máximo el } \\
\text { primer año }\end{array}$ & $\begin{array}{c}\text { Hasta la } \\
\text { actualidad }\end{array}$ & Total \\
\hline $\begin{array}{c}\text { Apoyo emocional pareja } \\
\text { durante gestación }\end{array}$ & NO & $70,0 \%$ & $12,5 \%$ & & $16,7 \%$ \\
\cline { 2 - 5 } Total $(\mathrm{N}=50)$ & $30,0 \%$ & $87,5 \%$ & $100,0 \%$ & $83,3 \%$ \\
\hline
\end{tabular}

Fuente: elaboración propia

En relación al AS material, la familia de origen es la red que provee este tipo de apoyo para una de cada dos universitarias $(53,1 \%)$. Una de cada tres mujeres informa apoyarse en la red formal-guarderías (30,6\%). En el $10,2 \%$ de ocasiones el apoyo material lo provee la dupla familia origenpropia (10,2\%). Sólo en el 4,1\% de casos asume la tarea exclusivamente la familia propia. Hay un $2 \%$ de universitarias que busca apoyo material en la red de amigos/as.

Por tanto, el apoyo material, es decir el cuidado del hijo/a de la estudiante, es asumido en gran medida por la red informal y más concretamente por la familia de origen de ella, especialmente por la abuela, como corroboran los hallazgos obtenidos.

Resulta sorprendente que cuatro de cada cinco informen de apoyo emocional de sus parejas cuando el apoyo material lo brinda la familia de origen. Podría indicar cierta idealización de la relación con su pareja que luego no se concreta en cuestiones prácticas y materiales 
de corresponsabilidad en la crianza o que apoyen la conciliación de la universidad y la maternidad.

En cuanto a apoyo económico, el 4,1\% de madres universitarias generan por sí mismas todos los recursos para su subsistencia y la de su hija/o. E1 2\% cuenta con una beca universitaria de 400 USD/semestre 8 . Otro 2\% recibe cuota de alimentos de la expareja para el sostenimiento de su hijo/a. La familia de origen provee a una de cada tres madres universitarias todo el apoyo monetario para estudios y maternidad (36,7\%) y en idéntica proporción lo hace la dupla familia origen y propia $(36,7 \%)$. La familia propia asume exclusivamente el apoyo económico en el 18,4\% de casos.

Aunque las mujeres informan percibir AS de diferentes tipos en porcentajes diferentes según la estructura de la red que provee apoyo, se ha encontrado evidencia de interrupción de estudios a causa de la maternidad. Estos hallazgos cambian sustancialmente según la situación de convivencia, y vuelven a emerger dos perfiles diferenciados que se describen a continuación.

1. Madres universitarias solteras: Abandonan menos la universidad a causa de la maternidad pero lo hacen por periodos más largos. Informan menos estructuras de apoyo y mayores dificultades para obtener AS económico. Utilizan más la red de apoyo formal (guarderías) para obtener apoyo material (cuidado).

Tres de cada cinco universitarias solteras no abandonaron sus estudios a causa de la maternidad $(66,7 \%)$ pero entre quienes sí suspendieron los estudios, la gran mayoría lo hizo por períodos prolongados superiores a tres semestres (85\%). Casi dos de cada cinco asume la crianza en solitario desde la noticia de su embarazo $(38,1 \%)$, por tanto sin apoyo emocional, material o económico de la pareja.

Entre las solteras que sí informan apoyo emocional de la pareja, se percibe un descenso conforme avanza el tiempo del embarazo al período de puerperio e inicio de la maternidad. Si tres de cada cinco se sintió acompañada por su pareja durante el embarazo (61,9\%), al periodo de puerperio el abandono de la responsabilidad paterna llegaba al 47,6\%. El 19\% fue acompañada hasta como máximo el primer año de nacido el/

${ }^{8}$ Que solo puede solicitarse a partir del $2^{\circ}$ año de carrera.

Prospectiv a. Revista de Trabajo Social e inter vención social No. 27, enero-junio 2019: pp. 107-137@)(ङ) 
la bebé y sólo uno de cada tres parejas acompaña en la crianza hasta la actualidad (33,3\%).

El AS de tipo emocional en este conjunto de madres universitarias proviene, en seis de cada diez casos, de su familia de origen (61,9\%). La pareja actual empata con el porcentaje de apoyo emocional que se identifica en la red informal de amistades: $14,3 \%$.

En el AS de tipo material, es la familia de origen quien cuida a la hija/o de la estudiante en más de uno de cada dos casos $(57,1 \%)$ y generalmente lo hace la abuela. La segunda estructura de apoyo informada corresponde a la red formal-guardería, que provee cuidados en uno de cada tres casos $(33,3 \%)$.

El AS de tipo económico es percibido $(66,7 \%)$ de su familia de origen. El 23,8\% informan percibir apoyo tanto de su familia de origen como del padre de su hijo/a. (Empata porcentualmente) En este grupo se ubican todas aquellas universitarias que asumen en solitario además de la crianza, la manutención económica del hijo/a y de sí mismas $(4,8 \%)$ y en idéntico porcentaje se reportan los casos de quienes cuentan con la beca económica proveniente de la red formal-universidad (4,8\%).

2. Madres universitarias en convivencia (en pareja): Abandonan más la universidad a causa de la doble condición madre-esposa pero lo hacen por periodos más cortos; informan más estructuras de apoyo pero la familia de origen sigue siendo la principal estructura que provisiona apoyo funcional. Son más acompañadas por sus parejas en embarazo y crianza (pero no en todos los casos).

En el grupo de madres universitarias en convivencia en pareja se encuentra más abandono de los estudios respecto a las solteras. Dos de cada tres sí interrumpió su formación a causa de la maternidad $(66,7 \%)$. Sin embargo estas mujeres logran reincorporarse a sus estudios en menos tiempo, en comparación al otro grupo de solteras, pues se incorporan pasado uno, dos o tres semestres en idénticas proporciones $(33 \%$ en cada periodo indicado).

En relación al AS emocional todas fueron acompañadas por la pareja durante el embarazo, pero al año de crianza, el 14,3\% habían sido abandonadas por su pareja. El análisis detallado de las variables sociales 
de este grupo, permitió determinar que, en uno de cada tres casos, el matrimonio ha sido una exigencia social que no ha ido acompañado de la corresponsabilidad masculina en la crianza. Otras fuentes que proveen apoyo emocional durante la crianza son la dupla familia de origen-propia $(53,6 \%)$, la familia de origen $(17,9 \%)$, la familia propia $(14,3 \%)$ y las amistades (14,3\%).

El AS de tipo material lo suministra la familia de origen a una de cada dos universitarias de este grupo (50\%), seguida por la ayuda de la dupla familia origen-propia (14,3\%). La familia extensa de la pareja, aporta un $4,8 \%$ de este tipo específico de apoyo. La red formal-guardería se informó en un $28,6 \%$ de los casos. Por tanto la familia de origen y concretamente la abuela, es la principal cuidadora.

En cuanto al AS de tipo económico, la mayor provisión de este apoyo es suministrado por la dupla familia de origen-propia (46,4\%); en segundo lugar, la familia propia $(32,1 \%)$ y en un $14,3 \%$ sólo la familia de origen. El $3,6 \%$ de ellas, aporta en exclusiva al sustento financiero de su hogar.

\subsection{Percepción de apoyo social formal desde la universidad.}

Lo primero que emerge del estudio es la existencia de indicadores que revelarían falta de apoyo social percibido por las madres estudiantes proveniente de la estructura de apoyo formal-universidad e incluso rechazo/ discriminación por parte de la institución y sus agentes. Las situaciones percibidas como discriminatorias dentro de la universidad y a causa de la maternidad, varían en función de la interrupción o no de los estudios, y también, según se pertenezca al grupo de solteras o mujeres en convivencia de pareja, como se presenta en la tabla 4. 
Tabla 4. Interrumpir estudios por maternidad*Tipos de discriminación percibidas en la universidad tabulación cruzada

\begin{tabular}{|c|c|c|c|c|c|}
\hline \multicolumn{6}{|c|}{$\%$ dentro de Interrumpir estudios por maternidad } \\
\hline \multirow{2}{*}{\multicolumn{2}{|c|}{ Convivencia actual }} & \multicolumn{3}{|c|}{$\begin{array}{c}\text { Tipos de discriminación percibidas en la } \\
\text { universidad }\end{array}$} & \multirow{2}{*}{ Total } \\
\hline & & $\begin{array}{l}\text { No AS } \\
\text { emocional }\end{array}$ & $\begin{array}{c}\text { No facilitar } \\
\text { conciliación } \\
\text { de ambos }\end{array}$ & $\begin{array}{l}\text { Minusvalorar } \\
\text { su rendimiento } \\
\text { académico }\end{array}$ & \\
\hline \multirow{3}{*}{$\begin{array}{c}\text { Sin } \\
\text { convivencia } \\
\text { actual } \\
\text { (soltera) }\end{array}$} & \multirow{2}{*}{\begin{tabular}{cc} 
Interrumpir & $\mathrm{NC}$ \\
\cline { 3 - 3 } estudios por & $\mathrm{SI}$ \\
maternidad &
\end{tabular}} & $40,0 \%$ & $40,0 \%$ & $20,0 \%$ & $100,0 \%$ \\
\hline & & & $66,7 \%$ & $33,3 \%$ & $100,0 \%$ \\
\hline & Total $(\mathrm{N}=21)$ & $25,0 \%$ & $50,0 \%$ & $25,0 \%$ & $100,0 \%$ \\
\hline \multirow{3}{*}{$\begin{array}{l}\text { Convivencia } \\
\text { actual } \\
\text { (casada o } \\
\text { unión libre) }\end{array}$} & \multirow{2}{*}{$\begin{array}{ll}\text { Interrumpir } & \mathrm{N} \\
\text { estudios por } & \mathrm{S} \\
\text { maternidad }\end{array}$} & $100,0 \%$ & & & $100,0 \%$ \\
\hline & & $66,7 \%$ & $33,3 \%$ & & $100,0 \%$ \\
\hline & Total $(\mathrm{N}=28)$ & $80,0 \%$ & $20,0 \%$ & & $100,0 \%$ \\
\hline
\end{tabular}

Fuente: elaboración propia

En esta dimensión, las respuestas obtenidas mediante preguntas abiertas, se agruparon en tres categorías, conforme la revisión de la literatura: No percibir apoyo emocional, no facilitar la conciliación de ambos roles y minusvalorar el rendimiento académico. En la categoría "no percibir apoyo emocional" se han incluido las respuestas relacionadas con bromas o indirectas, comentarios hirientes, incomprensión por parte de compañeros y docentes del ejercicio del doble rol. La segunda dimensión denominada "no facilitar la conciliación" agrupa la exclusión de actividades académicas, negativas del/a docente de ingreso al aula con su hija/o; humillación por ingreso a clase con hijos/as, comentarios de que una madre no puede ser estudiante, etc.

Finalmente, la categoría "minusvaloración del rendimiento académico" agrupa respuestas relativas a que el/a docente consideraba que la estudiante ya no realizaba trabajos con esfuerzo, al trato diferente hacia ella después de su embarazo y maternidad, a la manifestación sutil por parte de la/el docente de que la estudiante, quien antes fuera valorada como estudiante sobresaliente, ahora fuera tratada como una estudiante del promedio. 
En el grupo de madres universitarias que nunca interrumpieron estudios a causa de la maternidad, se encontró que siete de cada diez (70\%) percibió alguna discriminación en la universidad. Y en el $60 \%$ de ocasiones percibió más de una forma de discriminación. Respecto a las manifestaciones percibidas de discriminación, el 44,4\% se sintió discriminada por algún/a docente a causa de la maternidad; también se identificó la dificultad para conciliar ambos roles y la falta de apoyo emocional en dos de cada cinco casos ( $40 \%$ respectivamente).

Finalmente, dentro del grupo de las universitarias que sí abandonaron los estudios a causa de la maternidad, el 68,2\% de ellas percibió alguna discriminación al retornar a la universidad. Pero ellas a cambio del grupo de universitarias que nunca abandonó, en su mayoría sólo percibieron una única forma de discriminación (66,7\%). La manifestación informada con mayor prevalencia fue la dificultad para la conciliación de ambos roles madre y universitaria (50\%). En este grupo el 31,8\% de universitarias sintió discriminación por un/a docente a razón de su maternidad.

\section{Discusión}

El ejercicio de la maternidad se asume como una situación devenida y obligada, que impacta de manera profunda en la vida de la mujer y es vivida desde dos extremos: o asumen en solitario la crianza como madres solteras, lo que en el Ecuador conlleva sufrir el fuerte estigma social que le acompaña, o se ven abocadas a casarse como consecuencia del embarazo. Pasando así de ejercer el rol de estudiante al triple rol de universitaria, madre y esposa, con el consiguiente incremento de mayores dificultades de conciliación, en coincidencia con los hallazgos del estudio colombiano con madres jóvenes de Micolta-León (2007).

La doble condición de madre y universitaria, supone una confrontación entre el imaginario social de la maternidad con las demandas de tiempo, esfuerzo y dedicación, y las mismas demandas que también requiere la universidad. Las jóvenes se ven abocadas a intentar conciliar ambos roles, siempre mediadas por el estereotipo buenas-malas madres que impone la presión social (Amantze, 2015; Castañeda-Letelier, 2015; Cimino-Sandoval 
et al., 2014; Lozano-Cabezas et al., 2016). Varios estudios en diferentes países han documentado que, como resultado de la maternidad, se produce un cambio en su estilo de vida y un retraso en sus estudios que llega incluso a determinar la deserción escolar. El riesgo de abandono es mayor cuando ésta coincide con los primeros semestres tal como se ha probado en la literatura científica (Cimino-Sandoval et al., 2014; Domínguez-Aguirre et al., 2015; Estupiñán-Aponte y Rodríguez-Barreto, 2009; EstupiñánAponte y Vela, 2012; Lozano-Cabezas et al., 2016; Miller y Arvizu, 2016).

La situación de convivencia se convierte en una condición diferenciadora de la trayectoria vital de las estudiantes. Las solteras perciben sobre todo dificultades para conciliar ambos roles, lo que puede ser consecuencia de su mayor permanencia en el sistema educativo a pesar de la maternidad indeseada y las menores fuentes de apoyo con las que cuentan. Para ellas, el AS constituye uno de los principales factores que facilitan la permanencia en la universidad y se explica en gran medida por el papel que asume la red familiar, sobre todo la madre de la estudiante, en la provisión de apoyos específicos que facilitan la conciliación del doble rol en su condición de madres solteras. Las universitarias que conviven en pareja son las que más abandonan la universidad, lo que podría estar hablando de presiones sociales para la prevalencia del rol de madre-esposa; sin embargo, se reinsertan a sus estudios en periodos más cortos respecto de las solteras, e informan más fuentes de AS. En uno u otro caso, la conciliación entre estudios y crianza tiene matices muy diferentes. La maternidad se antepone como rol superior, conforme a las costumbres y prácticas tradicionales en ambos casos, siendo este tema coincidente con otra investigación de Chile, donde las universitarias, también supeditaron su formación a las exigencias de la maternidad (Cimino-Sandoval et al., 2014) y con referencias similares a otros estudios de la región (Domínguez-Aguirre et al., 2015; EstupiñánAponte y Rodríguez-Barreto, 2009; Estupiñán-Aponte y Vela, 2012; Lozano et al., 2016; Zapata-Posada et al., 2016).

Por otra parte, y en relación al apoyo emocional de la pareja, la evidencia estadísticamente significativa de que los padres que no estuvieron apoyando durante el embarazo, tampoco lo hicieron durante la crianza, y que el porcentaje de abandonos aumenta conforme avanza el crecimiento 
de la hija/o, está en línea con los descubrimientos de Estupiñán-Aponte y Rodríguez-Barreto (2009) en otra investigación similar con universitarias embarazadas en el contexto colombiano.

Entre universitarias solteras, las fuentes de apoyo son menores respecto a quienes conviven en pareja. En las primeras, hay muchos más hogares que son monoparentales $(66,7 \%)$, familias más numerosas, con peores condiciones de vivienda y menor nivel socioeconómico, aspectos que pueden influir en la capacidad de la red para proveer apoyos específicos, lo cual coincide con la necesidad de buscar más apoyo para el cuidado en otras fuentes, o incluso, carecer de algunos tipos de apoyo, por ejemplo, el económico.

En el caso de las universitarias unidas en pareja, la evidencia estadísticamente significativa entre matrimonio por embarazo y convivencia actual, se relaciona con el cumplimiento del mandato cultural del ideal de madre-esposa, supeditando cualquier proyecto vital al ejercicio del rol superior de "madre", que se hace latente en las mayores cifras de abandono temporal de los estudios. El hallazgo de que las universitarias se casan porque se embarazan, también aporta evidencia empírica relacionada con la vigencia y legitimación de un modelo hegemónico y dominante de familia patriarcal. El descubrimiento coincide con un estudio reciente del Trabajo Social en México, donde se encontraron evidencias de la vigencia de estereotipos de género en estudiantes universitarias (Rodríguez-Otero y Mancinas-Espinoza, 2016) y otra investigación en Argentina en la que los roles del ámbito doméstico incidían en la represión de las ambiciones femeninas (Cristiani et al., 2015).

Sin embargo, resulta curioso que una de cada tres universitarias viva con su familia de origen, aún adquiriendo el estatus social de mujer casada; legitimando culturalmente el nacimiento de su hijo/a conforme los mandatos sociales, pero sin que por ello se incremente la corresponsabilidad masculina esperada en la convivencia en pareja (ya que viven separados y ella con la familia de origen).

Finalmente, las madres universitarias solteras percibieron más discriminación en la universidad respecto a las que informaron convivencia en pareja y las manifestaciones fueron diferentes en uno y otro grupo. Los 
tipos de discriminación identificados coinciden con los hallazgos de otra investigación de Colombia (Estupiñán-Aponte y Vela, 2012), quienes también encontraron referencias relativas a la falta de apoyo emocional y la minusvaloración del rendimiento académico entre madres universitarias (aunque sin discriminar por tipos de criterio sus hallazgos), diferencias que emergen de este estudio, tal y como aquí se presenta.

\section{Conclusiones}

Este estudio da cuenta de la condición de la maternidad indeseada de las mujeres universitarias de la sierra ecuatoriana de cuyo análisis se concluye que:

Es alarmante la prevalencia de embarazos en jóvenes ecuatorianas universitarias pues una de cada tres mujeres entre 18 y 19 años es madre. Este hecho social con una tendencia en incremento, influye en las posibilidades de desarrollo de las mujeres y sus hijos e hijas. Los resultados de esta investigación encontraron un altísimo porcentaje de embarazos indeseados pero llevados a término (80\%); temor en las mujeres a revelar su condición $(62 \%)$ y pensamientos de aborto en uno de cada cinco casos $(24,5 \%)$; por tanto la maternidad es un suceso repentino que interrumpe el proyecto académico y las posibilidades de desarrollo intelectual y profesional de las mujeres jóvenes. Existe evidencia estadísticamente significativa de que las parejas que no estuvieron apoyando durante el embarazo, tampoco lo hicieron durante la crianza, y que el porcentaje de abandonos aumenta conforme avanza el crecimiento de la hija/o.

Las condiciones sociodemográficas condicionan la posibilidad o no interrumpir los estudios universitarios a pesar de quedarse embarazada de forma indeseada. En este sentido emergen dos grupos claramente diferenciados: las solteras que llevan a cabo el proyecto de la maternidad en solitario, y las casadas o unidas en pareja que en general, aunque no en todos los casos, lo llevan en común con su pareja. Siendo las universitarias que viven con sus parejas quienes más abandonan sus estudios respecto a las solteras. 
La situación de convivencia en pareja se convierte, como se ha caracterizado, en una condición diferenciadora de la trayectoria vital de las estudiantes. Sin embargo, en el caso de las solteras, las deserciones son por períodos mucho más largos, lo que sugiere que en uno u otro caso, la conciliación entre estudios y crianza tiene matices muy diferentes.

El apoyo social estructural y funcional se evidencia como la variable que permite a las jóvenes llevar a término su embarazo, así como la terminación antes o después de sus estudios.

Desde el Trabajo Social con perspectiva de género: el altísimo porcentaje de embarazos no planificados y los proyectos de maternidad devenida como madres solteras o como esposas, son dos hallazgos que ponen el acento en:

- La evidencia de un tabú asociado a la planificación familiar que termina en maternidades indeseadas; es necesario orientar y separar el ejercicio de la sexualidad de la procreación como opción elegida.

- El segundo aspecto enfatiza la evidencia empírica encontrada sobre la plena vigencia del modelo de madre-esposa, conforme las expectativas sociales de la cultura patriarcal en la sierra ecuatoriana, con el consecuente incremento de roles domésticos. O en el otro caso, el abandono de las responsabilidades de crianza del padre y por tanto una maternidad asumida en solitario, el madresolterismo, con importantes estigmas sociales que producen discriminación social.

- El tercer aspecto retoma el profundo impacto que supone en la vida de las mujeres un embarazo indeseado pero llevado a término y las dificultades posteriores de desarrollo personal y profesional.

- El hecho de que universitarias solteras lleven a término embarazos no planificados, asuman la crianza en solitario en condiciones de mayor vulnerabilidad socioeconómica y que abandonen menos los estudios por esta causa, hace evidente la necesidad de apoyos específicos que atiendan a las demandas propias de conciliación del doble rol como una de las estrategias que facilite la permanencia en el sistema de educación superior. 
- La universidad como institución clave en la mejora de la calidad de vida, empoderamiento y progreso de las mujeres ecuatorianas, tendrá como reto diseñar conjuntamente con las madres universitarias programas institucionales y estrategias de conciliación que sean acordes a las necesidades reales que ellas presentan. En este sentido, es menester del Trabajo Social liderar este proceso y atender a una cuestión de género que impacta en el presente y futuro de estas mujeres con el objetivo de minimizar el riesgo de deserción universitaria y reducir el altísimo índice de embarazos indeseados en la universidad.

- Asumir la crianza del hijo/a siendo una joven en proceso formativo, sin experiencia laboral y con dependencia económica, como revela el perfil mayoritario que emerge de este estudio, hace, por tanto, aún más difícil la conciliación del doble rol e incrementa el riesgo de abandono de los estudios.

- En este sentido, las madres universitarias en general y las madres solteras universitarias en particular, constituyen un colectivo preferente para el desarrollo de una política regional y/o institucional que prevenga la deserción escolar. Esta investigación demuestra que las madres universitarias abandonan sus estudios por largos periodos, lo que a su reingreso, podría dificultar retomar el ritmo de las actividades académicas, aumentando el riesgo de abandono.

En resumen, y como se mencionó anteriormente, esta investigación da cuenta de la condición de maternidad indeseada de las mujeres universitarias de la sierra ecuatoriana según el AS percibido (desde las perspectivas estructural y funcional) y las variables sociodemográficas que las afectan. Se comparó al grupo de mujeres solteras con el grupo de mujeres en convivencia en pareja (casadas o en unión libre) al ser ésta variable la que emergió como diferenciadora de las desigualdades encontradas. Se recomienda replicar la investigación en otros contextos ecuatorianos, o latinoamericanos, con muestras mayores y/o en otras áreas de conocimiento para identificar posibles diferencias o similitudes con 
Johanna Alexandra Reina-Barret o-Cristina Alexandra Criollo-Espín - Karina Fernández-D’Andrea

otras poblaciones de madres universitarias y conocer el fenómeno con mayor profundidad.

\section{Referencias bibliográficas}

Amantze, S. (2015). "Subversivas": Malas madres" y familias "desnaturalizadas". Cuadernos pagu, (44), 423-452.

Arvelo, L. (2004). Maternidad y paternidad, género y psicoanálisis. Otras Miradas, 4(2), 92-98.

Barrera, M. (1986). Distinctions between social support concepts, measures, and models. American Journal of Community Psychology, 14(4), 413-445.

Barrón, A. (1996). El apoyo social. Aspectos teóricos y aplicaciones. Torrejón de Ardoz. Madrid: Siglo Veintiuno Editores.

Castañeda-Letelier, M.F. (2015). Ser estudiantes, madres y padres una dualidad cotidiana. (Tesis de pregrado). Universidad de Chile. Facultad de Ciencias Sociales. Antropología social. Santiago, Chile.

Castillo-Sánchez, A. (2015). La práctica social de la maternidad y de la paternidad en jóvenes estudiantes de nivel superior: un acercamiento a las problemáticas cotidianas enfrentadas durante la vida académica. Estudios sobre las Culturas Contemporáneas, 21(2), 103-123.

CEPAL. (sf). Indicador CELPASTAT. Maternidad en adolescentes: Porcentaje de mujeres adolescentes de 15-19 años de edad que son madres. País Ecuador. Años 1984-2012. Edad 18-19 años. Recuperado de http://interwp.cepal.org/ sisgen/ConsultaIntegrada.asp?idIndicador $=2187 \&$ idioma $=$ e.

Cimino-Sandoval, J., Durán-Sepúlveda, B., Herbage-Amaro, R., Palma-Jara, M., y Roa-Vergara, J. (2014). Ser madre y estudiante universitaria en la Universidad de Santiago de Chile. Un estudio exploratorio acerca de las implicaciones psicosociales en el enfrentamiento de ambos roles. Revista de estudios cualitativos USACH, 1(1), 23-39.

Cristiani, L.S., Bertolotto, M., Anderson, M., Polinelli, S.N., Collavini M., Seijo, F y Delfino, M. (2015). Significado cultural de la crianza y de los roles materno y paterno. Aportes a la reflexión teórica. En VI Congreso Internacional de Investigación y Práctica Profesional en Psicología XXI Jornadas de Investigación Décimo Encuentro de Investigadores en Psicología del MERCOSUR. Universidad de Buenos Aires. Facultad de Psicología. Buenos Aires, Argentina.

Díaz, P. (1987). Evaluación del apoyo social. En R. Fernández-Ballesteros (Coord). El Ambiente. Análisis psicológico (pp. 125-149). Madrid: Ediciones Pirámide. 
Domínguez-Aguirre, G.A., Martínez-Peralta, A., Herrera-Barragán, H y RincónAguirre, M.E. (2015). Impacto personal y escolar del embarazo y maternidad en estudiantes universitarios. Investigación y práctica en psicología del desarrollo, 1, 167-173.

Estupiñán-Aponte, M. y Rodríguez-Barreto, L. (2009). Aspectos psicosociales en universitarias embarazadas. Revista Salud Pública, 11(6), 988-998.

Estupiñan-Aponte, M. y Vela, D. (2012). Calidad de vida de madres adolescentes estudiantes universitarias. Revista Colombiana de Psiquiatría, 41(3), 536-549.

Facio, A. (2002). Engenerando nuestras perspectivas. Otras Miradas, 2(2), 49-79.

Feldman, L., Goncalves, L., Chacón-Puignau, G., Zaragoza, J., Bagés. N y Pablo, J. de. (2008). Relaciones entre estrés académico, apoyo social, salud mental y rendimiento académico en estudiantes universitarios venezolanos, Universitas Psychologica, 7(3), 739-751.

Ferrer-Pérez, V., y Bosch-Fiol, E. (2013). Del Amor Romántico a La Violencia de Género. Para una coeducación emocional en la Agenda Educativa. Profesorado. Revista de Currículum y Formación de Profesorado. 17(1), 105-22.

García-Selgas, F. y Casado-Aparicio, E. (2010). Violencia en la pareja: Género y vínculo. Madrid: Talasa.

Guzmán-Gómez, C. (2017). Las nuevas figuras estudiantiles y los múltiples sentidos de los estudios universitarios. Revista de la educación superior, 46(182), 71-87.

Hombrados-Mendieta, I. (2013). Manual de psicología comunitaria. Madrid: Ed. Síntesis.

INE. (2018). Indicadores demográficos básicos. Recuperado de http://www.ine. es/dyngs/INEbase/es/operacion.htm?c=Estadistica_C\&cid=1254736177003\& menu $=$ ultiDatos\&idp $=1254735573002$.

INEC. (sf). Proyecciones poblacionales. Proyección provincias, sexos y áreas 2010-2020. Disponible en: http://www.ecuadorencifras.gob.ec/ proyecciones-poblacionales/.

INEC. (2017). Nacimientos y defunciones. Recuperado de http://www. ecuadorencifras.gob.ec/nacimientos_y_defunciones/.

Lagarde, M. (1990). Identidad Femenina. Recuperado de https://xenero.webs. uvigo.es/profesorado/purificacion_mayobre/identidad.pdf.

Lozano-Cabezas, I., Iglesias-Martínez, M.J. y Alonso-Sanz, A. (2016). Conciliación estudiantil-familiar: un estudio cualitativo sobre las limitaciones que afectan a madres Universitarias. Arbor, 192(780). doi: 10.3989/arbor.2016.780n4017.

Maeso-Fernández, M.E., Salamanca-Castro, A.B., Sánchez-Castro, S., GilPascual, J.A., Amezcua- Sánchez, A., y Ayuso-Medina, N. (2015). Nivel de sexismo ambivalente en estudiantes de primer curso de educación secundaria 
obligatoria de la ciudad de Madrid. Journal of feminist Gender and Women Studies, (2), 23-31.

Micolta-León, A. (2007). Componentes básicos para la atención psicosocial a padres y madres adolescentes. Prospectiva. Revista de Trabajo Social e intervención social, (12), 207-224. Doi: 10.25100/prts.v0i12.961.

Miller, D., y Arvizu, V. (2016) Ser madre y estudiante. Una exploración de las características de universitarias con hijos y breves notas para su estudio. Revista de la Educación Superior, 1(177), 17-42.

Montes de Oca, V. (2000). Relaciones Familiares y redes sociales. En V. Montes de Oca (Ed.), Envejecimiento demográfico en México: retos y perspectivas (pp.289-326). México: Consejo Nacional de Población.

Mora-Bleda, E. (2013). El paradigma género y mujeres en la historia del tiempo presente. Revista Historia Autónoma, (2), 143-160.

Observatorio de Igualdad de Género de América Latina y el Caribe. (sf). Maternidad en adolescentes. América Latina, el Caribe y la Península Ibérica (22 países): Mujeres adolescentes de 15 a 19 años de edad que son madres, último año disponible (en porcentajes). Recuperado de https://oig.cepal.org/ es/indicadores/maternidad-adolescentes.

Pacheco-Carpio, C.R., Cabrera-Albert, J.S., Mazón-Hernández, M., GonzálezLópez, I., y Bosque-Cruz, M. (2014). Estereotipos de Género Sexistas. Un estudio en jóvenes universitarios cubanos de medicina. Revista de Ciencias Médicas, 18(5), 853-867.

Palomar-Verea, C. (2004). "Malas Madres": La construcción social de la maternidad. Debate Feminista, 30, 12-34.

Paz, J.J. y Cepeda, M. (2002). La Revolución Juliana: nación, ejército y bancocracia. Ecuador: Abya-yala.

Pizani, M. (2009). Los derechos humanos de las mujeres en el Ecuador. En Programa Andino de Derechos Humanos, PADH (Comp.) ¿Estado constitucional de derechos?: informe sobre derechos humanos Ecuador 2009 (pp. 449-463). Quito: Universidad Andina Simón Bolívar; Abya Yala.

Puyana-Villamizar, Y. y Mosquera-Rosero, C.P. (2005). Traer "hijos e hijas al mundo": significados culturales de la paternidad y la maternidad. Revista Latinoamericana de Ciencias Sociales, Niñez y Juventud, 3(2), 1-21.

Preciado-Cortés, F., Acuña-Cepeda, M.E., y García-Rivera, M.E. (2005). Repercusiones del embarazo y la maternidad en la vida académica de las estudiantes universitarias. Géneros, 13(36), 125-132.

Rodríguez-Otero, L.M., y Mancinas-Espinoza, S.E. (2016). Nivel de sexismo y estereotipos de género en estudiantes de Trabajo Social Regiomontanas. Azarbe. Revista Internacional De Trabajo Social y Bienestar, (5), 17-30. 
Sánchez-Benítez, N. (2016). La experiencia de la maternidad en mujeres feministas. Revista Nómadas, (44), 255-267.

Texis, M., y Jiménez, M. (septiembre, 2016). El impacto de los problemas de género en la trayectoria educativa de las universitarias. En Congreso Internacional de Educación. Debates en Evaluación y Currículum. Universidad Autónoma de Tlaxcala. Tlax, México

Zapata-Posada, J.J., Castro-Rodelo, Y.Y., y Agudelo-Bedoya, M.E. (2016). Abuelas antes de lo esperado: cambios, participación en la crianza y relaciones intergeneracionales. Prospectiva. Revista de Trabajo Social e intervención social, (22), 117-140. doi: 10.25100/prts.v0i22.1239.

Cómo citar:

Reina-Barreto, J.A., Criollo-Espín, C.A. y Fernández-D’Andrea, K. (2019). El apoyo social en la maternidad indeseada de estudiantes universitarias ecuatorianas: Análisis desde la perspectiva de género. Prospectiva. Revista de Trabajo Social e intervención social, (27), 107-137. Doi: 10.25100/prts. v0i27.6745. 